RGB photoelasticity applied to the analysis of membrane residual stress in glass

This article has been downloaded from IOPscience. Please scroll down to see the full text article.

2012 Meas. Sci. Technol. 23025601

(http://iopscience.iop.org/0957-0233/23/2/025601)

View the table of contents for this issue, or go to the journal homepage for more

Download details:

IP Address: 2.158.246.155

The article was downloaded on 18/12/2011 at $15: 30$

Please note that terms and conditions apply. 


\title{
RGB photoelasticity applied to the analysis of membrane residual stress in glass
}

\author{
A Ajovalasit, G Petrucci and M Scafidi \\ University of Palermo, Viale delle Scienze, 90128 Palermo, Italy \\ E-mail: a.ajovalasit@gmail.com, giovanni.petrucci@unipa.it and michele.scafidi@unipa.it
}

Received 21 June 2011, in final form 13 November 2011

Published 16 December 2011

Online at stacks.iop.org/MST/23/025601

\begin{abstract}
The measurement of residual stresses is of great relevance in the glass industry. The analysis of residual stress in glass is usually made by photoelastic methods because glass is a photoelastic material. This paper considers the determination of membrane residual stresses in glass plates by automatic digital photoelasticity in white light (RGB photoelasticity). The proposed method is applied to the analysis of membrane residual stresses in some tempered glass. The proposed method can effectively replace manual methods based on the use of white light, which are currently provided by some technical standards.
\end{abstract}

Keywords: residual stresses, glass, RGB photoelasticity, image processing

(Some figures in this article are in colour only in the electronic version)

\section{Introduction}

It is known that photoelasticity can be used for the analysis of residual stresses in glass $[1,2]$ since it is a photoelastic material. The development of digital photoelasticity [3, 4] allows the user to automate the analysis of residual stress in glass as shown in $[5,6]$ in the case of the phase-shifting photoelastic method.

This paper, further developing the results presented in [6], concerns the analysis of membrane residual stresses in glass plates by the use of RGB photoelasticity in white light. Classical photoelasticity in white light has been widely used for the analysis of residual stress in glass. Specifically, the determination of the retardation has been carried out through the analysis of the colors by means of the tint plate (fullwave plate) $[7,8]$ and also by means of standard strain discs $[1,2,9,10]$. The mere observation of the colors and the use of a chart of colors $[1,11]$, such as the one used in the technique of birefringent coatings, produce results that often have only a qualitative value. In this paper, the automation of photoelasticity in white light applied to the analysis of membrane residual stresses in glass plates by the use of RGB photoelasticity [12-14] is proposed. Then, the paper shows the application of RGB photoelasticity to the determination of membrane residual stresses in some tempered glasses, in particular a household shelf and the rear window of a car. The results obtained with the proposed method are compared with the results obtained by the phase-shifting method.

\section{Theoretical analysis}

In RGB photoelasticity, the model is usually observed by a dark field circular polariscope. Using monochromatic light, the intensity emerging from this polariscope is

$$
I=I_{0} \sin ^{2} \pi \delta
$$

where $I_{0}$ is the intensity of the monochromatic light source and, in two-dimensional photoelasticity, the retardation $\delta$ is related to the difference of principal stresses $\sigma_{1}-\sigma_{2}$ by the known relationship

$$
\delta=\frac{C_{\lambda} d}{\lambda}\left(\sigma_{1}-\sigma_{2}\right),
$$

where $d$ is the thickness of the photoelastic model and $C_{\lambda}$ is the photoelastic constant at wavelength $\lambda$ of the monochromatic light. The dark lines visible on the model, where the retardation $\delta$ is constant, are called isochromatic fringes. 
The retardation $\delta$ depends on the wavelength $\lambda$ and on the dispersion of birefringence according to equation (2) which provides

$$
\delta=\delta_{0} \frac{\lambda_{0}}{\lambda} \frac{C_{\lambda}}{C_{0}}
$$

where $\delta_{0}$ is the retardation at the reference wavelength $\lambda_{0}$ and the term $C_{\lambda} / C_{0}$ takes into account the birefringence dispersion of the photoelastic material $[11,15,16]$.

In RGB photoelasticity, the isochromatic fringes are acquired in white light by a RGB camera that decomposes the image into three primary colors, red $(R)$, green $(G)$ and blue $(B)$, by means of three wide band filters. Due to the use of broadband filters, it is not possible to use the classical equation (1) of the dark field circular polariscope in monochromatic light. Not considering the background noise, the light intensity acquired by the RGB camera is indeed expressed $[12,14]$ by the following relationship:

$$
\begin{aligned}
I_{w j}= & \frac{1}{\lambda_{j 2}-\lambda_{j 1}} \int_{\lambda_{j 1}}^{\lambda_{j 2}} I_{0}(\lambda) T(\lambda) F_{j}(\lambda) \sin ^{2}\left(\pi \delta_{0} \frac{\lambda_{0}}{\lambda} \frac{C_{\lambda}}{C_{0}}\right) \\
& \times\left(1-\cos ^{2} 2 \alpha \sin ^{2} \varepsilon\right) \mathrm{d} \lambda \quad(j=R, G, B),
\end{aligned}
$$

where $I_{0}(\lambda)$ is the light intensity which depends on the characteristics of the light source, $T(\lambda)$ is the transmittance that depends on the spectral response of the tested material (glass), $F_{j}(\lambda)(j=R, G, B)$ are the spectral responses of the three camera filters, $\lambda_{j 1}$ and $\lambda_{j 2}$ are the lower and upper limits of the spectral response of the $j$ filter and the subscript $w$ indicates the use of white light, $\alpha$ is the angle which defines the direction of the maximum principal stress and $\varepsilon$ is the error that affects the retardation of the quarter-wave plates.

The determination of the retardation $\delta_{0}$ using equation (4) is not easy; therefore, the retardation is determined by using a database search approach. RGB photoelasticity allows the user to determine retardations up to, at least, three fringe orders using normal incandescent lamps [12]. In cases where the source is a fluorescent lamp having a discrete emission spectrum (common energy saving light bulbs for domestic use), it is possible to detect much higher orders [17], at least up to 12 fringe orders as shown in [14].

\subsection{Calibration procedure}

The classical calibration procedure [12] consists of acquiring the RGB values at each pixel along the transverse symmetry section of a calibration specimen subjected to bending. Each $R_{i}, G_{i}$ and $B_{i}$ triplet is stored in a calibration table (look-up table: LUT), similar to the color chart in use in the method of birefringent coatings, and is associated with a retardation value $\delta_{0 i}$. Typically a set of RGB triplets are acquired, corresponding to retardations increasing linearly from a point where the retardation is zero $\left(\delta_{0}=0\right)$ to a point subject to a maximum known retardation $\left(\delta_{0}=\delta_{0 N}\right)$. In general, the material of the calibration specimen must match the material used in the test. Otherwise, it is necessary to take into account both the different spectrum of colors (involved in the term $T(\lambda)$ that appears in equation (4)) and the different dispersion of birefringence. The effect of color variation between calibration and tested materials can be corrected by suitably modifying the LUT as described in [18]. The dispersion of birefringence is considered in the appendix, where it is shown that its effect is generally small (errors less than 0.05 fringe orders) for glasses with normal $\left(C_{\lambda}\right.$ decreases with increasing $\left.\lambda\right)$ dispersion of birefringence and retardations lower than three orders of those usually occurring in the case of residual stress in glass. Since the realization of a glass calibration specimen in bending is not simple in some instances, in this paper an alternative selfcalibration procedure, defined briefly Self-Cal, is proposed. The Self-Cal procedure consists in creating the LUT using a glass equivalent to that to be analyzed, subject to residual stresses not lower than those to be measured. Unlike the case of specimen subjected to bending, the retardation along the section where the RGB values are acquired must be determined by an independent method, such as the phase-shifting method [19]. Furthermore, the retardation is generally not linearly varying along the section itself, so that consecutive elements in the LUT do not correspond to a constant variation of retardation.

It is possible to take this feature into account in two ways, whose advantages will be explained in the following section.

- The first one consists in storing the retardation evaluated by the phase-shifting method in the calibration table along with the RGB triplets (correspondence LUT).

- The second one consists in creating a LUT with a linear relationship between the indices $i$ and the retardation $\delta_{0 i}$, by a proper interpolation of the acquired $R, G$ and $B$ values (linearized LUT).

The interpolation procedure, used in the linearized LUT, consists of the following steps:

(1) choice of the constant variation of retardation between two consecutive elements of the LUT $\delta_{s}$ (it is appropriate to use a value close to the minimum variation directly obtained in the acquisition step);

(2) determination of the values of the retardations $\delta_{0 i}$ in the range $0 \leqslant \delta_{0 i} \leqslant \delta_{0 N}$, spaced by the constant variation $\delta_{s}$ and

(3) determination of the corresponding levels $R_{i}, G_{i}$ and $B_{i}$, interpolating the experimental values (as an example by using a cubic spline or techniques like that proposed in [20]).

\subsection{Search for retardation}

In the analysis stage the $R, G$ and $B$ levels at points where the retardation is unknown are acquired. Each $R, G$ and $B$ triplet is then compared with the triplets $R_{i}, G_{i}$ and $B_{i}$ stored in the calibration table by means of an error function defined as

$$
e_{i}=\sqrt{\left(R_{i}-R\right)^{2}+\left(G_{i}-G\right)^{2}+\left(B_{i}-B\right)^{2}} .
$$

In each pixel, the index $i$ of the LUT that minimizes the error function (5) is determined.

Using the correspondence LUT, the retardation can be read at the position indicated by the index $i$, while using 
the linearized LUT the retardation can be calculated by the relationship

$$
\delta_{0 i}=\delta_{0 N} \frac{i}{N}, \quad 0 \leqslant i \leqslant N,
$$

where $\delta_{0 N}$ is the maximum retardation of the calibration table and $N$ is the index of the last element in the calibration table (corresponding to $\delta_{0 N}$ ).

The described procedure can be applied by evaluating the error function (5) for all the elements of the LUT ( $i=0,1$, $2, \ldots, N)$, or for a small portion of it; this second option is preferable [14]. In this case, considering the linearized LUT, for each $R, G$ and $B$ triplet to be analyzed, the range of the index $i$ in equation (5) is

$$
\left(i_{\delta_{s}}-\Delta i\right) \leqslant i \leqslant\left(i_{\delta_{s}}+\Delta i\right)
$$

where $i_{\delta s}$ is the index of the LUT corresponding to the correct value of the retardation in the adjacent pixels to those being examined, and $\Delta i$ is an appropriate neighborhood given by [14]

$$
\Delta i=0.4 \frac{N}{\delta_{0 N}}
$$

This procedure, as well as having significantly less computational time, implicitly contains the condition of continuity of the retardation. It reduces the errors similarly to the procedures which use explicitly the condition of continuity of the retardation $[17,21]$. In this case the linearized LUT is more suitable, because it allows one to use equations (7) and (8) in a more straightforward manner; therefore, in this paper the linearized LUT was used.

\subsection{Determination of the stresses}

Once the retardation $\delta_{0}$ is determined, the difference of principal stresses is evaluated by equation (2) written for the reference wavelength reference $\lambda_{0}$, which provides

$$
\sigma_{1}-\sigma_{2}=\frac{\lambda_{0}}{C_{0} d} \delta_{0}
$$

where the photoelastic constant of glasses $C_{0}$ ranges, usually, between 2.4 and 3.6 Brewster ( 1 Brewster $\left.=1 \mathrm{TPa}^{-1}\right)$ [22].

In particular, the edges of flat glass sheet are usually compressed, and, due to boundary conditions, $\sigma_{y}=\sigma_{1}=$ 0 . Thus, the value of stress along the boundary is from equation (9)

$$
\sigma_{x}=\sigma_{2}=-\frac{\lambda_{0}}{C_{0} d} \delta_{0} .
$$

The stress normal to the contour $\left(\sigma_{y}\right)$ is nearly zero even near the boundary. So equation (10) applies until the point of inversion of the sign of the tension (identified by the presence of zero-order isochromatic fringe); after the fringe of order 0 , the tensile stress is again from equation (9)

$$
\sigma_{x}=\sigma_{1}=\frac{\lambda_{0}}{C_{0} d} \delta_{0}
$$

Equation (9) is always valid, while equations (10) and (11) are valid provided that the stress normal to the contour $\left(\sigma_{y}\right)$ is zero or nearly zero.

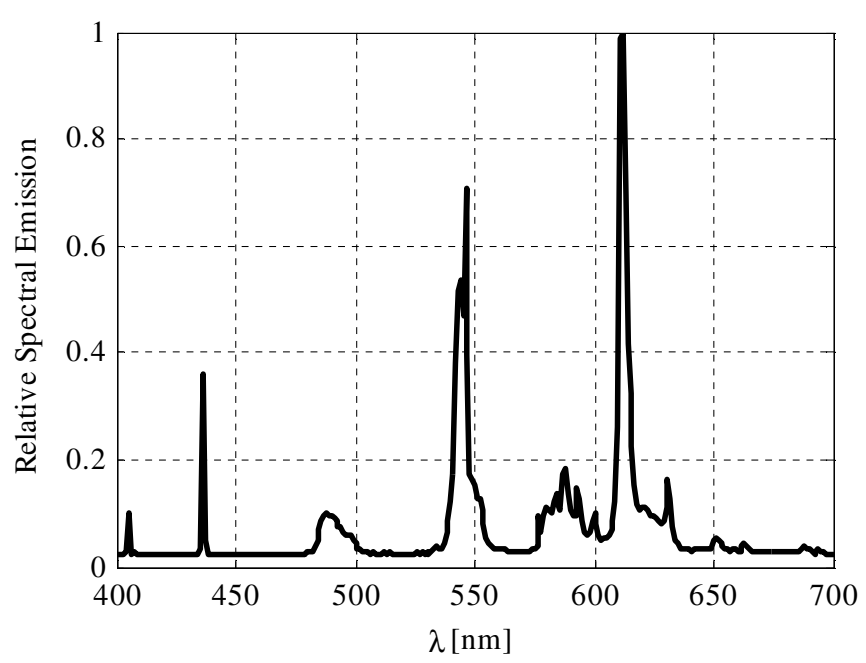

Figure 1. Emission spectrum of the white light source (common fluorescent tubes) used for the experiments.

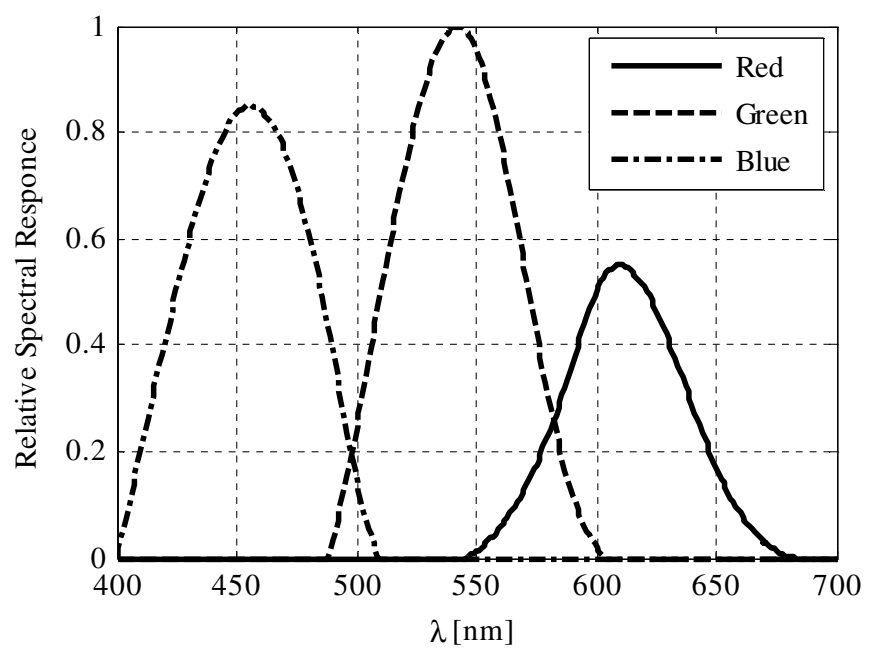

Figure 2. Approximate spectral distributions of filters $R, G$ and $B$ of the camera used for the experiments.

\section{Experiments}

The experiments were carried out using a dark field circular polariscope having quarter-wave plates corrected for the reference wavelength $\lambda_{0}=589 \mathrm{~nm}$ (monochromatic yellow light).

In order to obtain the best results using RGB photoelasticity, a fluorescent white light source with a discrete emission spectrum should be used [14, 17]. It is also important that the light source is uniform and has a high intensity in order to minimize the opening of the diaphragm of the optical system. For this reasons ten tubes (type: Philips Master 7L-D Super $8018 \mathrm{~W} / 827$ ) were used. Their spectrum, having three peaks at the wavelengths $\lambda_{R}=612 \mathrm{~nm}$ (red), $\lambda_{G}=546 \mathrm{~nm}$ (green) and $\lambda_{B}=436 \mathrm{~nm}$ (blue), is shown in figure 1 .

The acquisition system consists of a JVC KY-F30 3CCD RGB camera with the spectral responses shown in figure 2 and a Matrox-Meteor-II digital board having a spatial resolution of $768 \times 576$ pixels and a quantization of 256 RGB levels. 


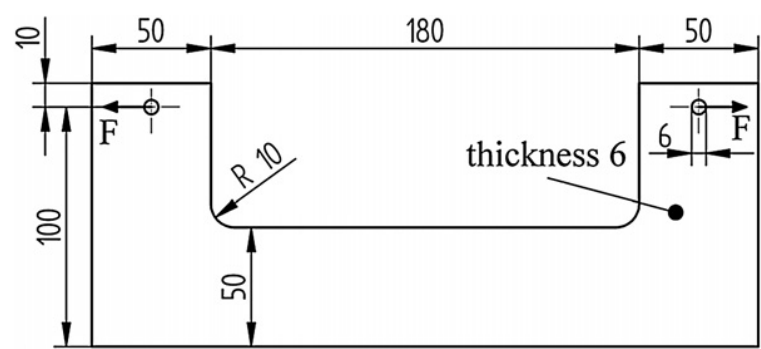

Figure 3. Calibration specimen (dimensions in $\mathrm{mm}$ ).

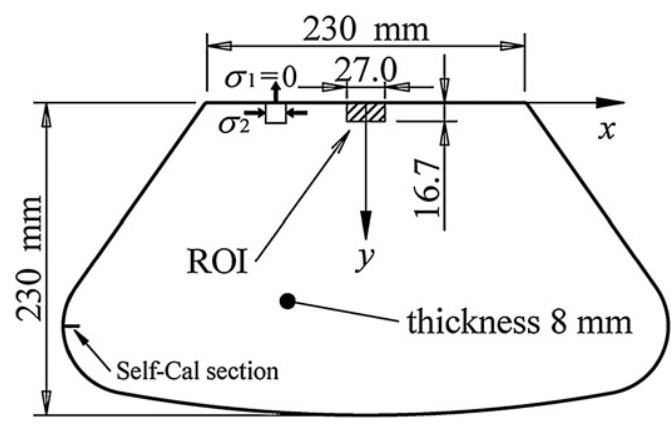

Figure 4. Shelf of tempered glass used for the tests.

Both resolution and quantization are good enough to obtain good results by the RGB analysis. The system allows the user to carry out a manual white balance, by which the RGB signals can be acquired in such a way that their maximum values are quite similar.

The camera is equipped with an optical system with manual setting of the diaphragm, of the focus and of the zoom; in the used system, a 1:1.4/7.5-97.5 zoom lens is actually mounted in series with a +2 diopter close-up lens. In any case, it is important that the magnification of the optical system allows the user to acquire images with a fringe gradient not higher than 0.1 orders/pixel [12]. In the experiments the optical system was adjusted in order to have a scale factor between 28.5 and 38.2 pixels $\mathrm{mm}^{-1}$.

The analysis was carried out on two tempered glass plates shown in figures 4 and $9(a)$. A polycarbonate (MM PSM1) calibration test model (figure 3) was also used.

The calibration was performed using both the polycarbonate specimen (figure 3) in which a maximum retardation $\delta_{0 N}=3$ fringe orders was produced and the same glasses, under test, using the self-calibration procedure described above.

As a first application example, figure 4 shows the shelf on which are indicated both the ROI (region of interest) and the section used for calibration. Figure 5 shows the isochromatic fringes at the section of self-calibration. Figure 6 shows the isochromatic fringes at the ROI and the section chosen for the measurement of the retardation.

Figure 7 shows the retardation $\delta_{0}$ along the measurement section determined by the RGB method (using both the calibration on polycarbonate and the self-calibration) and, for comparison, by the phase-shifting method based on six acquisitions [19].

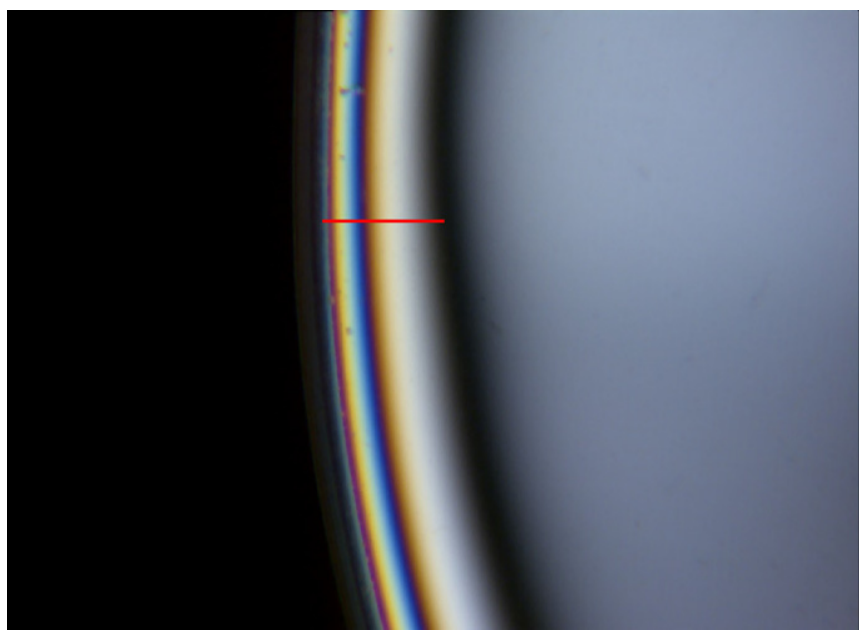

Figure 5. Shelf: isochromatic fringes. The red horizontal line denotes the section of the glass used for the self-calibration.

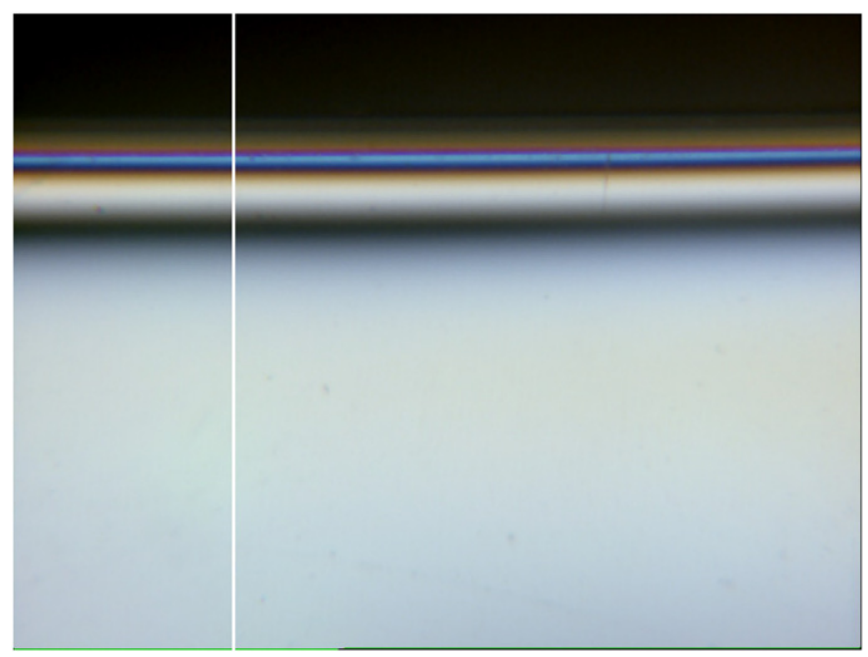

Figure 6. Shelf: isochromatic fringes at the ROI and measurement section of the retardation.

Similar results are obtained by the simplified phaseshifting method based on three acquisitions [5] and with the phase-shifting method in white light [16]. Near the rounded edge, the retardation is irregular and thus the graph has been truncated. In such a case, procedures are used to extrapolate the plot of fringe order at the boundary $[23,24]$. According to equation (9) and considering the factor $\lambda_{0} / C_{0} d$, the retardation $\delta_{0}$ represents the difference of the principal stresses. Figure 7 also shows the difference of the principal stresses referred to the mean value $C_{0}=3$ Brewster.

Figure 8 shows the error with respect to the phase-shifting method. That error, in the central area of the glass is between \pm 0.05 fringe orders, while in the area near the boundary reaches higher values. As a second example, figure 9 shows the rear window of a car with the indication of the ROI $(a)$ and the isochromatic fringes at the measurement section $(b)$.

Figure 10 shows the retardation $\delta_{0}$ along the measurement section determined by the RGB method (using both the calibration on polycarbonate and the self-calibration) and, for comparison, by the phase-shifting method based on six 


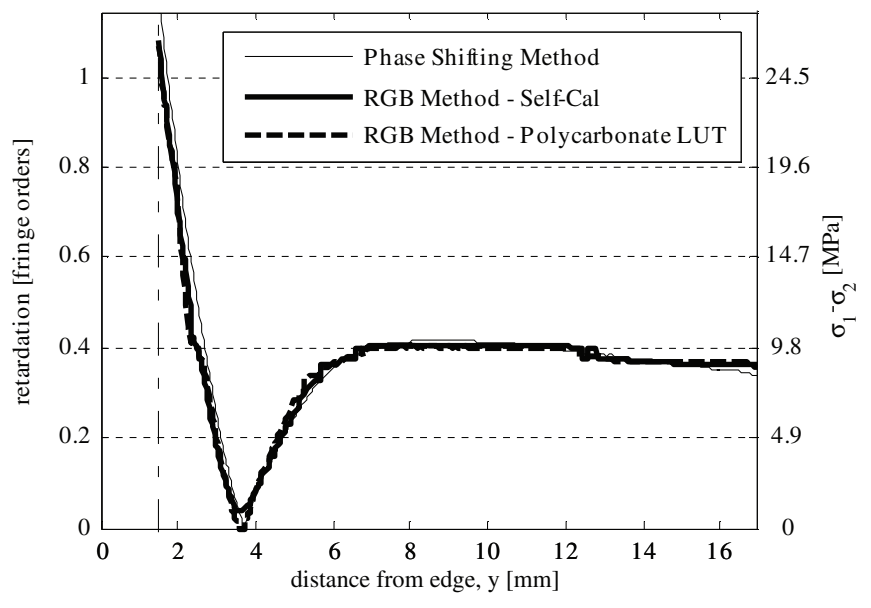

Figure 7. Shelf: retardation and difference of principal stresses $\left(\sigma_{1}-\sigma_{2}\right)$ along the measurement section determined by RGB photoelasticity (calibration on polycarbonate and the self-calibration) and by the phase-shifting method based on six acquisitions.

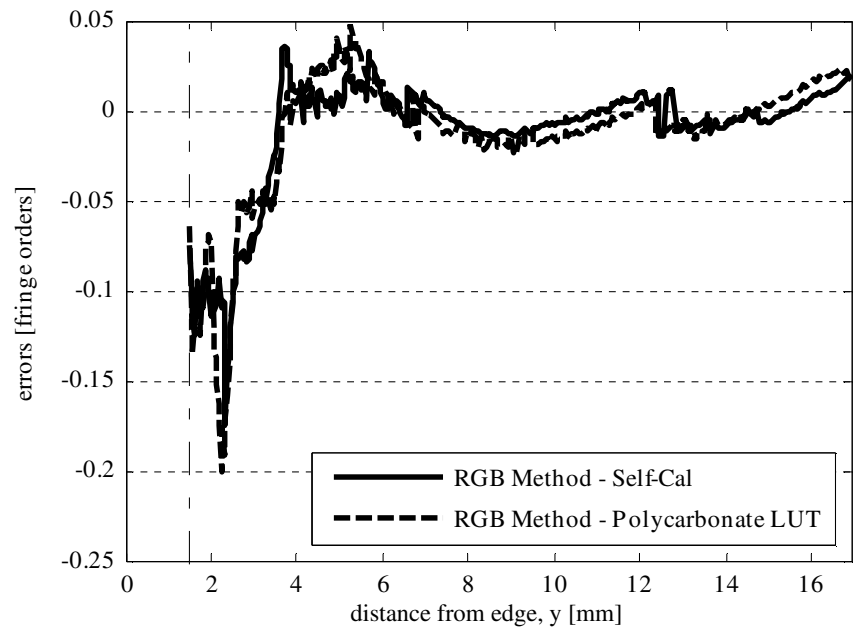

Figure 8. Shelf: error between the results obtained by RGB photoelasticity and the phase-shifting method based on six acquisitions.

acquisitions. The same figure shows the difference of the principal stresses again evaluated for the mean value $C_{0}=3$ Brewster.

Figure 11 shows the error with respect to the phaseshifting method based on six acquisitions. In this application this error, except for some isolated peaks, ranges between \pm 0.05 fringe orders.

For the glasses analyzed in this study, self-calibration and calibration on polycarbonate provide results that are equivalent in practice. The self-calibration procedure is generally preferable because it does not require the use of an auxiliary specimen made of a different material.

For both glasses, figure 12 shows the $\sigma_{x}$ stress values, evaluated with $C_{0}=3$ Brewster, along the $y$ direction. In particular, the stress $\sigma_{x}$ was evaluated by equations (10) and (11) that, as previously said, are valid near the boundary (about $10-15 \mathrm{~mm}$ in the $y$ direction as shown by figure 12) where the

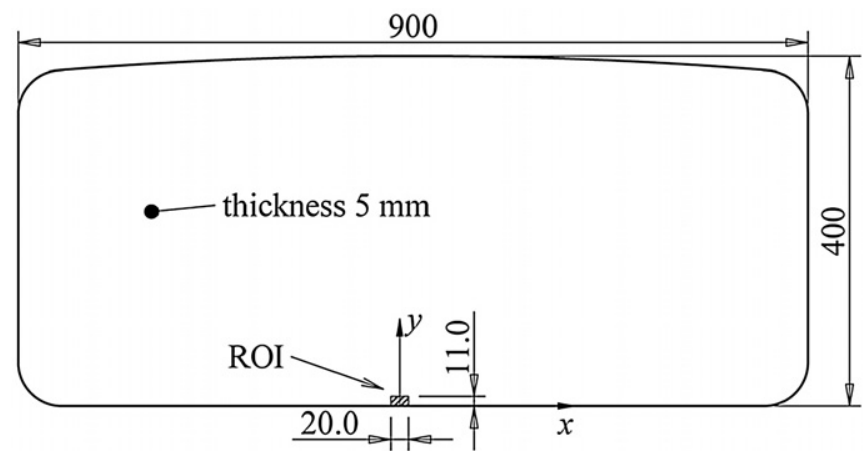

(a)

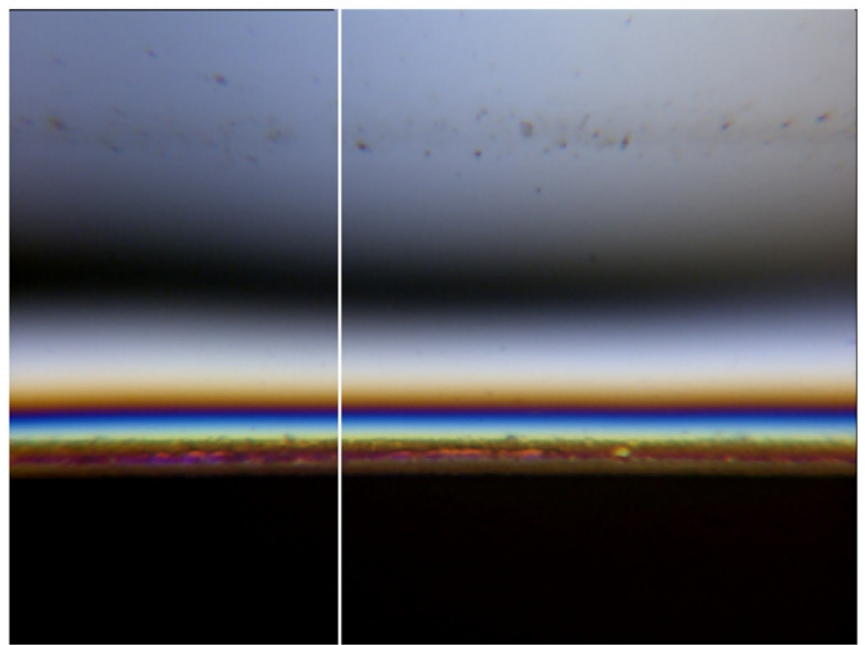

(b)

Figure 9. (a) Rear window of a car used for the analysis of residual stress, $(b)$ isochromatic fringes at the ROI and measurement section.

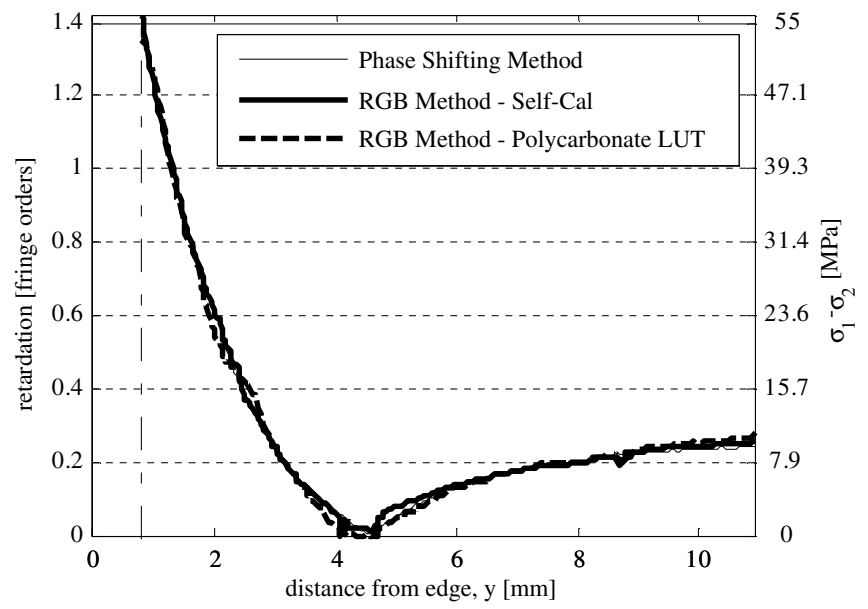

Figure 10. Rear window: retardation and difference of principal stresses $\left(\sigma_{1}-\sigma_{2}\right)$ along the measurement section determined by RGB photoelasticity (polycarbonate calibration and self-calibration) and the phase-shifting method based on six acquisitions.

stress normal to the contour $\left(\sigma_{y}\right)$ is zero or nearly zero. The trend of the $\sigma_{x}$ stress is similar to that shown in the literature for similar cases [25]. 


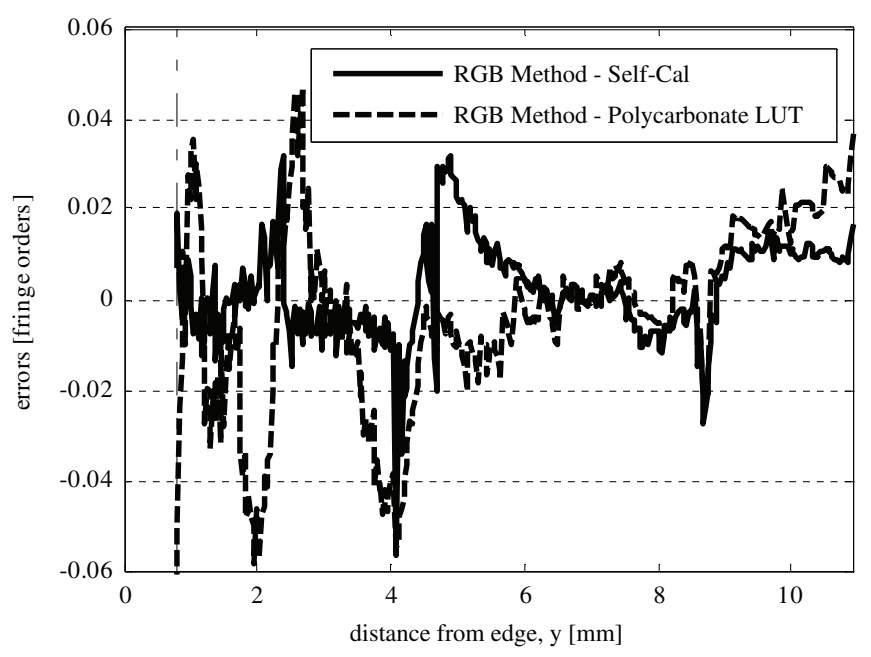

Figure 11. Rear window: error between the results obtained with RGB photoelasticity (polycarbonate calibration and auto-calibration) and the phase-shifting method based on six acquisitions.

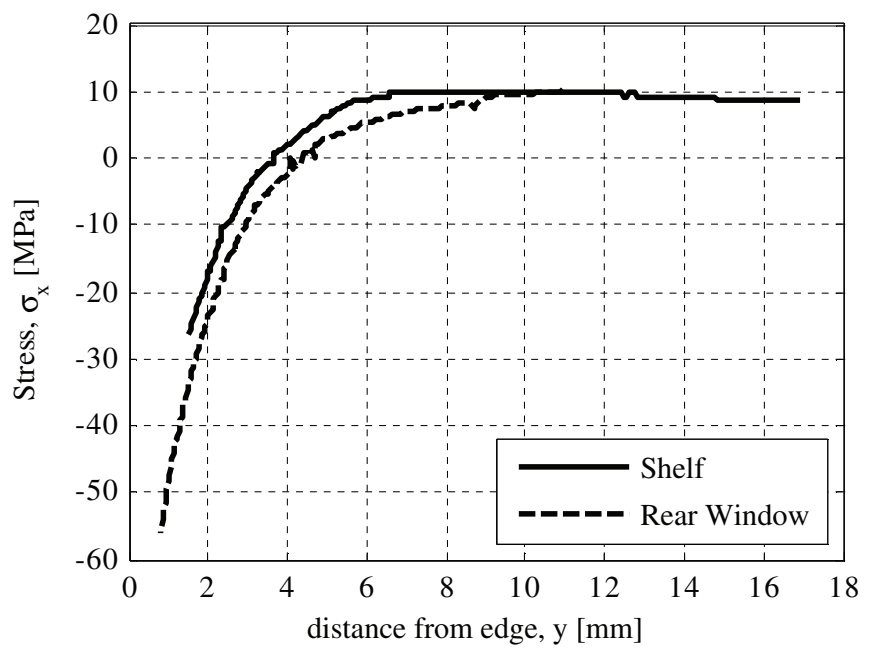

Figure 12. Stress $\sigma_{x}$ along the measurement sections based on the RGB Self-Cal retardation.

\section{Conclusions}

The research described in this paper concerns the determination of membrane residual stresses in glass plates by RGB photoelasticity.

The RGB method is here modified by the introduction of the self-calibration procedure, in order to avoid the use of an auxiliary calibration specimen and to take into account both the actual transmittance and the effective dispersion of the birefringence of the glass under consideration.

Apart from the images to be acquired for the calibration, the proposed technique requires the acquisition of only a single image of the glass to be analyzed, using the circular polariscope.

A comparison with the phase-shifting methods shows that the errors are usually within \pm 0.05 fringe orders. Despite the need for the calibration procedure, the proposed technique is easier and faster to apply than the traditional phase-shifting methods, which require from three to six acquisitions for each analysis. The ability of RGB photoelasticity to operate in real time with a single acquisition is therefore analogous to that achievable with the real-time phase-stepping polariscope recently proposed in [26].

In conclusion, the modified RGB photoelasticity technique proposed in this paper allows the user to automate the methods of membrane residual stress analysis in the glass provided by some technical standards. Due to its convenience and its relative simplicity, it is well suited to industrial applications for non-destructive testing of tempered glass.

\section{Appendix. Effect of dispersion of birefringence}

If the material used for calibration is different from that to be analyzed (glass), some error can be introduced. In fact, the two materials can differ in both the spectrum of the colors and dispersion of birefringence. This second aspect is considered below. Figure A1 shows the dispersion of birefringence of polycarbonate (MM PSM1), Araldite B [16] and some tempered glass [27, 28]. To examine the effect of birefringence dispersion, equation (4) is taken into consideration. This equation, neglecting the term due to the error of quarter-wave plates, becomes

$$
\begin{aligned}
I_{w j}= & \frac{1}{\lambda_{j 2}-\lambda_{j 1}} \int_{\lambda_{j 1}}^{\lambda_{j 2}} I_{0}(\lambda) T(\lambda) F_{j}(\lambda) \sin ^{2}\left(\pi \frac{\delta_{0} \lambda_{0}}{\lambda} \frac{C_{\lambda}}{C_{0}}\right) \mathrm{d} \lambda \\
& (j=R, G, B) .
\end{aligned}
$$

On the other hand, for the material used for the calibration (CAL), equation (4) gives

$$
\begin{aligned}
I_{w j}^{\mathrm{cal}} & =\frac{1}{\lambda_{j 2}-\lambda_{j 1}} \int_{\lambda_{j 1}}^{\lambda_{j 2}} I_{0}(\lambda) T^{\mathrm{cal}}(\lambda) F_{j}(\lambda) \\
& \times \sin ^{2}\left[\pi \frac{\delta_{0} \lambda_{0}}{\lambda}\left(\frac{C_{\lambda}}{C_{0}}\right)^{\mathrm{cal}}\right] \mathrm{d} \lambda \quad(j=R, G, B) .
\end{aligned}
$$

Comparison of equations (A.1) and (A.2) shows that, for a given retardation $\delta_{0}$, the signals acquired in both calibration and measurement steps are equal, i.e.

$$
I_{w j}=I_{w j}^{\mathrm{cal}} \quad(j=R, G, B),
$$

provided that the same material is used for both calibration and measurement in order to have the same spectrum of colors $\left[T^{\mathrm{cal}}(\lambda)=T(\lambda)\right]$ and the same dispersion of birefringence $\left[\left(C_{\lambda} / C_{0}\right)^{\mathrm{cal}}=\left(C_{\lambda} / C_{0}\right)\right]$.

The effect of the different spectra of colors is considered in the literature [18], while in the following the effect of dispersion of birefringence on the retardation obtained by the RGB method is considered. To this end, the $R, G$ and $B$ signals relative to both calibration and measurement were simulated and used to carry out the analysis of retardation. The simulation of the signals was carried out using equations (A.1) and (A.2), where $T^{\mathrm{cal}}(\lambda)=T(\lambda)=1$ for all materials, since only the effect of the dispersion of the birefringence is considered, and where

- $I_{0}(\lambda)$ is provided by the emission spectrum of fluorescent lamps (figure 1); 


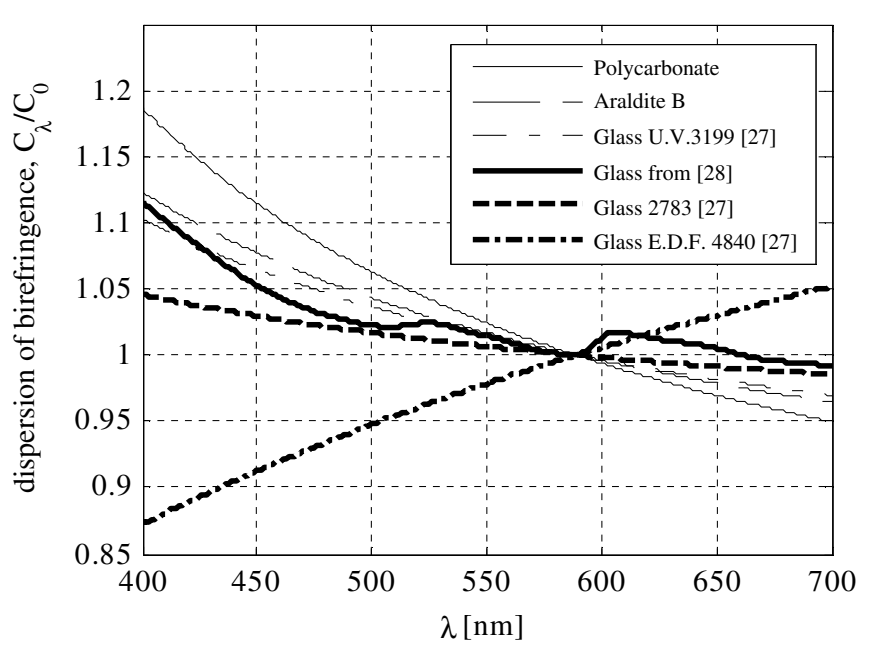

Figure A1. Dispersion of the birefringence of polycarbonate (MM PSM1), Araldite B and of some glasses.

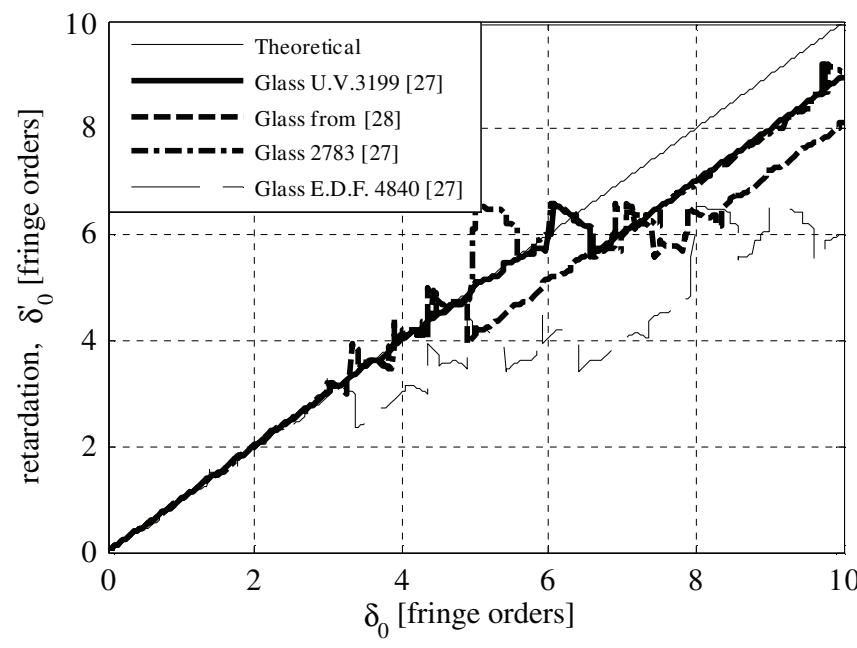

Figure A2. Retardation $\delta_{0}^{\prime}$ on the glasses of figure A1 as a function of the actual retardation $\delta_{0}$ using the calibration on polycarbonate.

- $F_{j}(\lambda)$ is provided by the spectral distribution of the filters $R, G$ and $B$ of the camera used (figure 2);

- $\delta_{0}$ is the retardation at the reference wavelength $\lambda_{0}$ and

- $\left(C_{\lambda} / C_{0}\right)^{\mathrm{cal}}$ and $C_{\lambda} / C_{0}$ indicate the dispersion of the birefringence of the particular material considered respectively during calibration and measurement phase (figure A1).

In particular, the effect of the dispersion of the birefringence on the retardation obtained by the RGB method was evaluated using the simulated calibration table relative to the polycarbonate.

Figure A2 shows just the retardation $\delta_{0}^{\prime}$ in the glasses of figure A1, determined by the above procedure, as a function of the actual retardation $\delta_{0}$. Figure A2 shows that the main effect of the dispersion of the birefringence is to reduce the maximum retardation that can be analyzed by RGB photoelasticity, from 12 fringe orders [14] to 6 fringe orders for the UV glass 3199 and to 3 fringe orders for the other glasses.

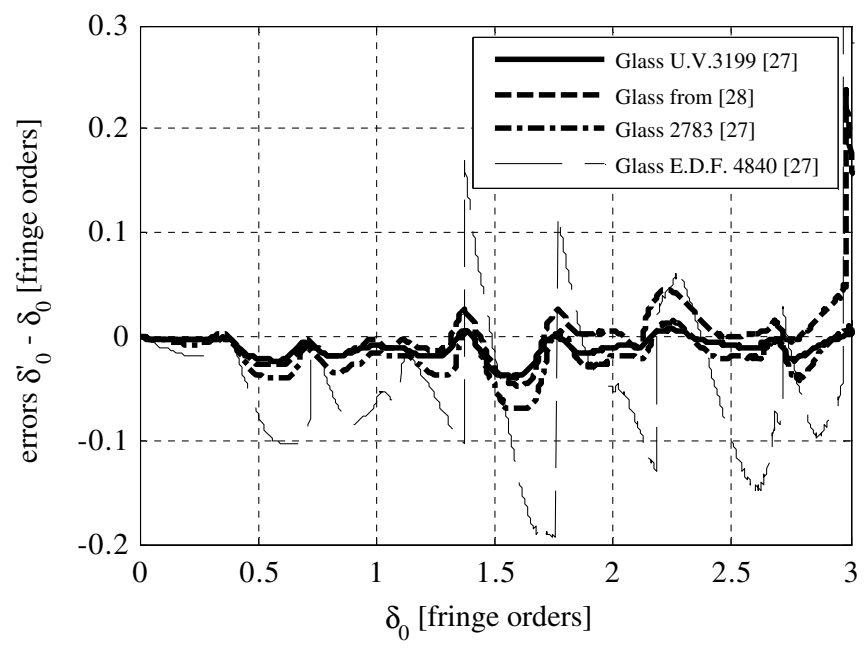

Figure A3. Glasses of figure A1: Errors $\delta_{0}^{\prime}-\delta_{0}$ as a function of the actual retardation $\delta_{0}$ using the calibration on polycarbonate.

In the following the analysis is limited to a maximum retardation of three fringe orders which is the field of interest in the analysis of membrane residual stress in the glass. Figure A3 shows the error that affects the retardation in the glasses shown in figure A1 evaluated from zero to three fringe orders.

The effect of the dispersion of the birefringence was also evaluated by using the simulated calibration table obtained on Araldite B and applying the RGB method to the glasses shown in figure A1. The results are similar to the previous ones but with minor errors with respect to the phase-shifting method. In fact by calibrating on polycarbonate and Araldite B, the error is always less than 0.05 and 0.03 (except for some isolated peak) fringe orders respectively for all the glasses in figure A1 (with the exception of glass EDF 4840). For glass EDF 4840 , which has a strong anomalous dispersion $\left(C_{\lambda}\right.$ increases with increasing $\lambda$ ), the error is less than 0.15 fringe orders. In conclusion, for the glasses which have normal dispersion shown in figure A1 and for retardations of less than three orders both the calibration obtained on polycarbonate (or Araldite) and the self-calibration can be applied. For the glass with anomalous dispersion, errors are higher and therefore only self-calibration is preferred.

\section{References}

[1] Aben H and Guillemet C 1993 Photoelasticity of Glass (Berlin: Springer)

[2] McKenzie H W and Hand R J 1999 Basic Optical Stress Measurement in Glass (Sheffield: Society of Glass Technology)

[3] Ramesh K 2000 Digital Photoelasticity (Berlin: Springer)

[4] Patterson E A 2002 Digital photoelasticity: principles, practice and potential Strain 38 27-39

[5] Ajovalasit A, Petrucci G and Scafidi M 2011 Measurement of edge residual stresses in glass by the phase shifting method Opt. Lasers Eng. 49 652-7

[6] Battaglia S, Ajovalasit A, Petrucci G and Scafidi M 2010 Analisi fotoelastica delle tensioni residue nel vetro Riv. Stazione Sperimentale Vetro 40 19-31 (in Italian) 
[7] ASTM 2005 Standard Method for Analyzing Stress in Glass Annual Book of ASTM Standards (West Conshohocken, PA: ASTM) ASTM F218

[8] ASTM C978 2009 Standard Test Method for Photoelastic Determination of Residual Stress in a Transparent Glass Matrix Using a Polarizing Microscope and Optical Retardation Compensation Procedures Annual Book of ASTM Standards (West Conshohocken, PA: ASTM)

[9] ASTM C148 2011 Standard Test Method for Polariscopic Examination of Glass Containers Annual Book of ASTM Standards (West Conshohocken, PA: ASTM)

[10] UNI 1997 Contenitori di vetro-Rilievo delle tensioni residue (in Italian) (Milano, Italy: UNI) UNI 7220

[11] Cloud G 1995 Optical Methods of Engineering Analysis (Cambridge: Cambridge University Press)

[12] Ajovalasit A, Barone S and Petrucci G 1995 Toward RGB photoelasticity_full field photoelasticity in white light Exp. Mech. 35 193-200

[13] Ramesh K and Deshmukh S S 1996 Three fringe photoelasticity - use of colour image processing hardware to automate ordering of isochromatics Strain 32 79-86

[14] Ajovalasit A, Petrucci G and Scafidi M 2010 RGB photoelasticity: review and improvements Strain 46 137-47

[15] Haake S J and Patterson E A 1993 The dispersion of birefringence in photoelastic materials Strain 29 3-7

[16] Ajovalasit A, Petrucci G and Scafidi M 2007 Phase shifting photoelasticity in white light Opt. Lasers Eng. 45 596-611

[17] Quiroga J A, Garcia-Botella A and Gomez-Pedrero J A 2002 Improved method for isochromatic demodulation by RGB calibration Appl. Opt. 41 3461-8
[18] Mandhu K R, Prasath R G R and Ramesh K 2007 Colour adaptation in three fringe photoelasticity Exp. Mech. 47 271-6

[19] Barone S, Burriesci G and Petrucci G 2002 Computer aided photoelasticity by an optimum phase stepping method Exp. Mech. 42 132-9

[20] Cleveland W S 1979 Robust locally weighted regression and smoothing scatterplots J. Am. Stat. Assoc. 74 829-36

[21] Madhu K R and Ramesh K 2007 Noise removal in three fringe photoelasticity by adaptive colour difference estimation Opt. Lasers Eng. 45 175-82

[22] Redner A S and Nickola W E 1984 Measurement of residual strains and stresses in transparent materials Exp. Tech. 8 29-32

[23] Redner A S and Voloshin A S 1990 Surface and face stress in tempered glass Proc. 9th Int. Conf. on Experimental Mechanics (Copenhagen) vol 2 pp 884-91

[24] ASTM C1279 2009 Standard Test Method for Non-Destructive Photoelastic Measurement of Edge and Surface Stresses in Annealed, Heat-Strengthened, and Fully Tempered Flat Glass Annual Book of ASTM Standards (West Conshohocken, PA: ASTM)

[25] Aben H, Anton J and Errapart A 2008 Modern photoelasticity for residual stress measurement in glass Strain 44 40-8

[26] Lesniak J, Zhang S J and Patterson E A 2004 Design and evaluation of the poleidoscope: a novel digital polariscope Exp. Mech. 44 128-35

[27] Coker E G and Filon L N G 1931 A Treatise on Photo-Elasticity (London: Cambridge University Press)

[28] Pindera J T and Sinha N K 1971 On the studies of residual stresses in glass plates Exp. Mech. 11 113-20 